\title{
e-Phaïstos
}

e-Phaïstos

Revue d'histoire des techniques / Journal of the history

of technology

V-1 2016 | 2018

Ville et technique

\section{Urbanisme et trame urbaine : ce que nous apprend l'histoire des villes}

Urban planning and urban patterns: what history tells us about the genesis of urban forms

Jean Laterrasse

\section{(2) OpenEdition}

Journals

Édition électronique

URL : https://journals.openedition.org/ephaistos/1281

DOI : 10.4000/ephaistos. 1281

ISSN : 2552-0741

Éditeur

IHMC - Institut d'histoire moderne et contemporaine (UMR 8066)

Référence électronique

Jean Laterrasse, "Urbanisme et trame urbaine : ce que nous apprend l'histoire des villes », e-Phaïstos

[En ligne], V-1 2016 | 2018, mis en ligne le 28 janvier 2018, consulté le 21 juin 2021. URL : http://

journals.openedition.org/ephaistos/1281 ; DOI : https://doi.org/10.4000/ephaistos.1281

Ce document a été généré automatiquement le 21 juin 2021.

Tous droits réservés 


\title{
Urbanisme et trame urbaine : ce que nous apprend l'histoire des villes
}

\author{
Urban planning and urban patterns: what history tells us about the genesis of \\ urban forms
}

Jean Laterrasse

1 Le mot urbanisme est récent et renvoie à l'époque contemporaine. Au sens strict, l'histoire de l'urbanisme, en tant que discipline, est limitée aux temps modernes. L'approche que nous proposons ici s'appuie sur l'histoire des villes et des pratiques associées à leur construction dans le temps long. Si on ne parlait pas d'urbanisme dans l'Antiquité ou au Moyen Âge, cela ne signifie pas pour autant que l'édification des villes n'obéissait pas à certaines règles, dont la lecture avec nos yeux d'aujourd'hui peut être tout à fait riche d'enseignements, à la fois pour comprendre la genèse des formes urbaines et pour orienter leurs évolutions.

\section{Les premières trames urbaines}

2 Dès l'Antiquité, deux principes émergèrent clairement dans les trames urbaines: le premier trouve sa concrétisation dans un schéma en damier, le second dans un schéma radio-centrique. Le schéma en damier, à l'instar du camp romain, privilégie une vision militaire, dictée notamment par une préoccupation de défense contre de potentielles attaques, et/ou de contrôle d'un territoire. Le schéma radio-centrique est le plus souvent l'apanage de congrégations religieuses, qui cherchent à reproduire dans l'organisation spatiale une vision de la société idéale (Pawlowski, 2002) (Figure 1 en annexe).

Ces deux formes s'illustrèrent particulièrement au Moyen Âge, dans l'opposition entre les bastides, où la trame en damier est de règle, et ce qu'on pourrait appeler un " urbanisme circulaire », remarquablement illustré en France par les "circulades» languedociennes. La répression des mouvements cathares imposa la forme des bastides comme forme dominante, mais les formes radio-centriques n'en restèrent pas moins 
longtemps présentes en France et dans de nombreux pays en Europe et en Orient. On en trouve encore l'empreinte dans de vieux centres urbains comme ceux de Montpellier, Sienne ou Milan.

4 Ainsi, les villes du Moyen Âge, loin du désordre spontané parfois décrit avec condescendance, procèdent d'un ordre élaboré. Les "circulades", marquées par un profond souci d'équité dans l'occupation des sols, en donnent une belle illustration : le centre y est généralement occupé par une tour et/ou un lieu de culte, et les parcelles soigneusement organisées en cercles concentriques, y sont délimitées par des voies de circulation radio-centriques. Dans cet agencement, la taille des parcelles augmente quand on s'éloigne du centre et vient compenser une moindre accessibilité. Ce principe remarquable d'organisation spatiale se trouve reproduit dans les zones rurales pour la conception des réseaux d'irrigation (Pawlowski, 2002). Les bastides, où apparaît l'organisation des parcelles et du réseau viaire en damier, procèdent à l'inverse d'une logique extensive, qui marque la prégnance des fonctions militaires et préfigure déjà la poussée de la ville hors de ses remparts. Une place centrale, d'abord dévolue aux fonctions logistiques, ménage un espace pour les fonctions commerciales (marchés) et pour les fonctions politiques ou sociales.

5 À la fin du Moyen Âge, les premiers plans urbains tel celui d'Amsterdam, témoignaient de la permanence et de l'intérêt des structures radio-centriques. Les premières règles d'urbanisme établirent une relation entre la hauteur des immeubles et la largeur des voies de circulation (Lavedan, 1982). À l'époque, la préoccupation principale était de se prémunir des risques de propagation des incendies de façade à façade, d'autant que le bois était un matériau essentiel pour la construction. Il est saisissant de constater que les règles alors édictées sont proches de celles qui prévalent aujourd'hui, du fait de la préoccupation de dimensionner les voies de circulation en relation avec les flux de déplacements que génère un bâti lus ou moins dense.

6 Lorsque, avec la Renaissance, la ville sortit de ses remparts et s'affranchit des contraintes spatiales antérieures, apparurent les places royales et les perspectives renouant avec l'héritage de la ville antique. Les trames urbaines, mieux mises en valeur, ne subirent pas alors de modifications essentielles. La véritable mutation urbaine et le changement d'échelle qui l'accompagna se produisirent avec la révolution industrielle, dans la seconde moitié du XVIII ${ }^{e}$ siècle et au XIX ${ }^{e}$ siècle.

\section{L'avènement de la fonction circulatoire}

7 Avec la concentration progressive de la production dans de grandes unités, la démographie urbaine augmenta rapidement au détriment de celle des zones rurales, et la bonne circulation des travailleurs ainsi que celle des marchandises devinrent une fonction urbaine essentielle. Des réseaux de transport public se constituèrent et se développèrent alors : à Paris, on ne comptait pas moins de onze compagnies d'omnibus qui, sous le Second Empire, de par la volonté de l'État, étaient regroupées en une compagnie unique, la Compagnie générale des omnibus (CGO). Dans la même période, l'ingénieur Loubat inventa le tramway, qui n'était autre, dans un premier temps, qu'un omnibus à traction hippomobile posé sur des rails de chemin de fer. Cette innovation permit de diminuer de manière importante les frottements des roues sur le sol (ils sont approximativement dix fois plus faibles) et donc de tracter des charges plus importantes, et aussi d'améliorer significativement le confort des passagers, mis à mal 
dans les omnibus traditionnels par l'état médiocre des chaussées pavées. Le métro de Londres, premier chemin de fer urbain utilisant pour partie des infrastructures souterraines, fut quant à lui inauguré en 1863. Ces nouveaux modes de transport urbain connurent un développement spectaculaire avec l'avènement dans les années 1880 de la traction électrique.

8 Mais ici, le progrès technique ne suffit pas. La question qui se posa très vite et de manière aiguë, fut celle de l'adaptation du tissu urbain à ce développement tout à fait nouveau des flux de personnes et de marchandises: l'émergence de cette fonction circulatoire, en passe de devenir une fonction urbaine vitale, était incompatible avec les plans d'urbanisme hérités du Moyen Âge. Deux personnages marquent alors profondément les mutations qui se produisirent dans l'agencement spatial des villes européennes : Ildefons Cerdà (1815 - 1876) à Barcelone et Georges Eugène Haussmann (1809 - 1891) à Paris. Beaucoup d'éléments différencient ces deux personnages, mais une chose au moins les rapproche : leur vision de la ville de l'ère industrielle, et la place accordée à la fonction circulatoire, au cœur de démarches de transformation urbaine de grande ampleur.

Cerdà a suivi une formation d'ingénieur des Ponts. Dans un contexte marqué par la destruction des murs d'enceinte de Barcelone (1859), il était chargé par le gouvernement espagnol de réaliser les plans d'expansion de la ville. L'objectif fixé pour ce projet fut de concevoir la nouvelle partie de la ville comme un lieu qui combine harmonieusement la fonction résidentielle et la fonction circulatoire, en opposition avec la vieille ville, congestionnée et propice aux épidémies. Le projet de Cerdà, l'Eixample, se montra novateur à la fois par sa vision d'ensemble et par plusieurs de ses caractéristiques: le plan est un plan en damier, censé permettre "à l'infini» l'extension urbaine par simple juxtaposition de nouvelles mailles. Deux grands boulevards en diagonale (un seul est finalement construit) drainent les flux transverses entre des points éloignés de la ville. Cette même préoccupation d'intégrer pleinement la fonction circulatoire amena Cerdà à imaginer des carrefours "à pans coupés " permettant une séparation des flux selon leur direction. La fonction résidentielle devait quant à elle être assurée par des îlots en $U$ ouverts sur des patios intérieurs et communiquant avec l'extérieur pour faciliter leur traversée piétonne (la pression foncière amena par la suite à construire sur les espaces réservés à ces patios). L'ensemble s'appuie sur des études très précises, où les références scientifiques sont nombreuses, qu'il s'agisse de la disposition géométrique des terrains, ou de l'orientation des rues censées faciliter la bonne ventilation de la ville. Ou encore de la dimension économique et sociale du projet, sa démarche étant marquée par une forte préoccupation de justice sociale. Au-delà de cette œuvre majeure, Cerdà s'imposa comme un des premiers théoriciens de l'urbanisme, au travers notamment de sa Teoría General de la Urbanización (Théorie générale d'urbanisation) publiée en 1867, et est salué à juste titre comme un des principaux précurseurs de l'urbanisme des réseaux, c'est-à-dire de l'intégration dans la conception urbaine des potentialités ouvertes par le développement des grands réseaux techniques (Dupuy, 1991).

Haussmann fut quant à lui, préfet de la Seine de 1853 à 1870 . À ce titre, il dirigea les transformations de Paris sous le Second Empire et mit en œuvre un vaste plan de rénovation. Au milieu du XIX siècle, malgré les travaux déjà diligentés par les prédécesseurs d'Haussmann tels Rambuteau, les rues de Paris, étroites, encombrées et insalubres, compromettaient sérieusement son développement économique. L'idée 
maîtresse des gigantesques travaux qu'il entreprit était de remédier à cette situation tout en améliorant significativement l'hygiène, en réaction aux épidémies qui sévissaient de manière récurrente (l'épidémie de choléra de 1832 a particulièrement marqué les parisiens). Le chantier était immense : on estime qu'en moins de vingt ans, sous l'impulsion d'Haussmann, ce sont près de $60 \%$ des immeubles qui furent démolis et reconstruits, et encore ce chiffre ne prend-il pas en compte les transformations réalisées dans le cadre de l'annexion des communes limitrophes.

Figure 2. L'Eixample, plan de 1859 du projet de Cerdà

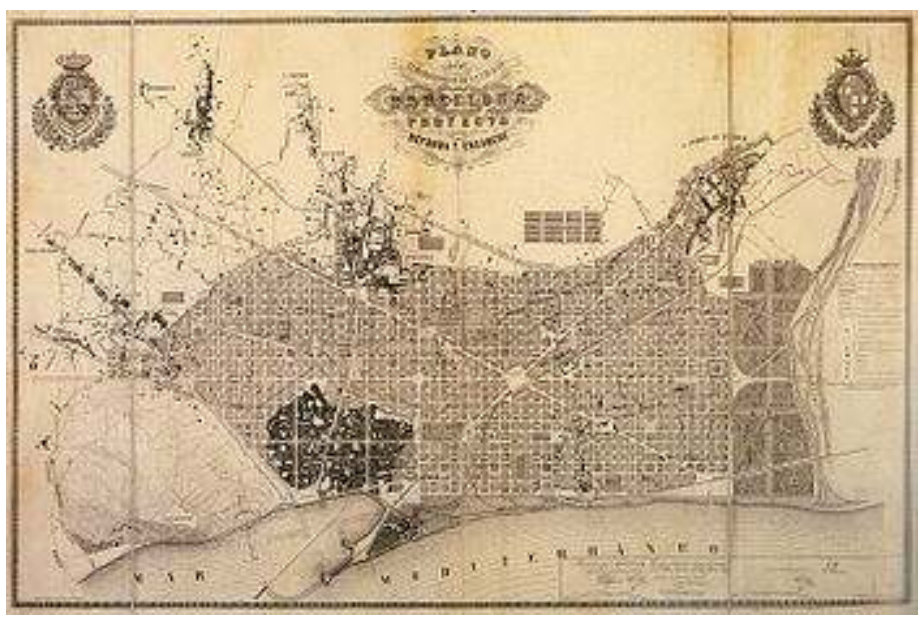

Figure 3. Les « percées » haussmanniennes entre 1850 et 1870

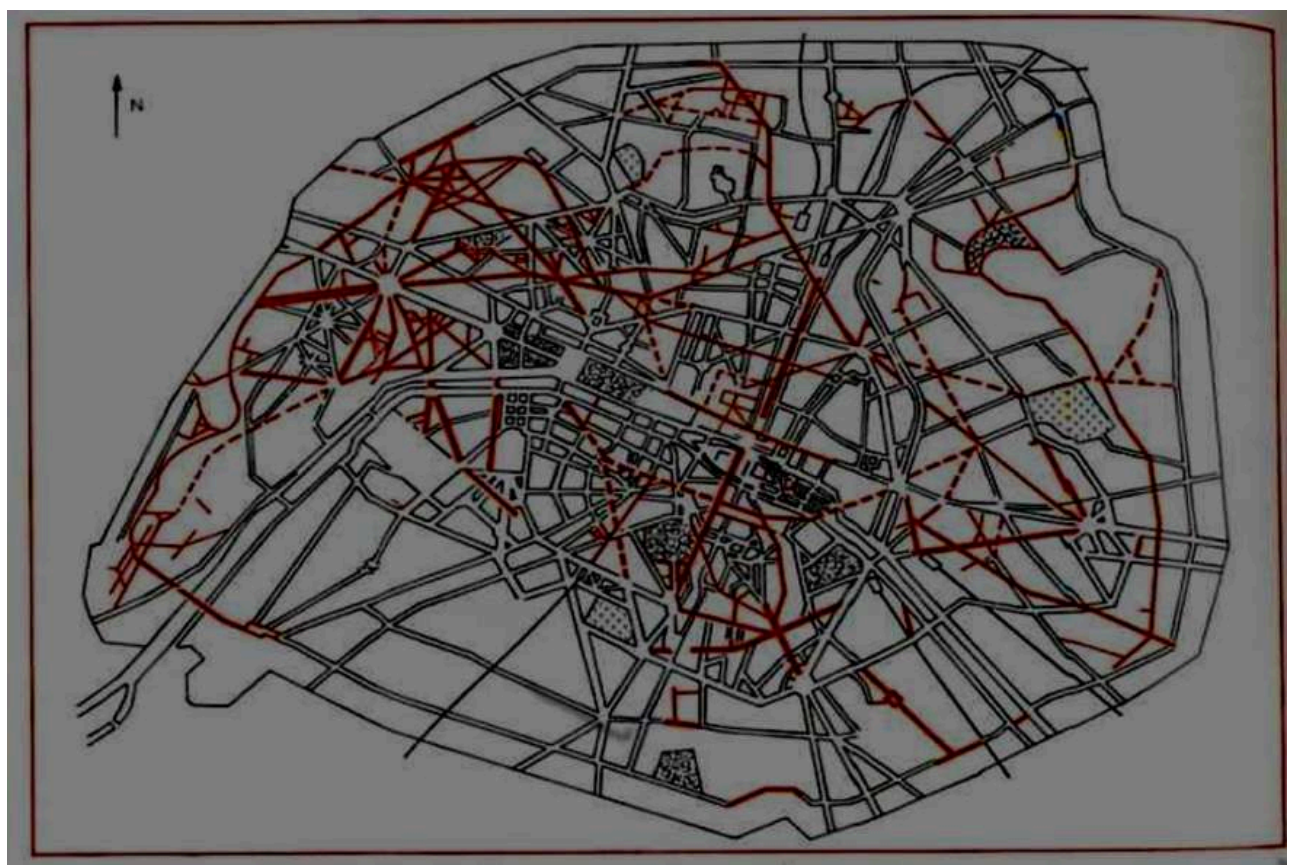

Contrairement à Cerdà, Haussmann n'était pas un théoricien. Son approche était beaucoup plus pragmatique; mais comme Cerdà, sa vision d'une grande métropole moderne est portée par la préoccupation d'articuler la fonction circulatoire, désormais essentielle pour l'activité économique, avec les autres fonctions urbaines. Son obsession de la ligne droite a de manière polémique été assimilée par ses détracteurs à 
la seule préoccupation du maintien de l'ordre. Tout porte à penser que, si il n'avait pas la même fibre sociale que Cerdà et s'il a assumé la gentrification de Paris, l'ambition de son plan allait très au-delà de la gestion des épisodes de tension politique. En dix-huit ans, des boulevards et avenues sont réalisés de la place du Trône (actuelle place de la Nation) à la place de l'Étoile, et de la gare de l'Est à l'Observatoire (au sud de Paris) et à la place d'Enfer (aujourd'hui place Denfert-Rochereau). Les percées qu'il dessina dans la trame urbaine sont également marquées par un souci permanent de mettre en valeur les monuments anciens et nouveaux, et ce même souci d'embellissement se manifesta par l'aménagement de nombreux parcs et jardins, tels le parc Montsouris ou celui des Buttes Chaumont. Des règles strictes furent fixées aux nouveaux immeubles construits en bordure des boulevards, qui se devaient de respecter l'ordonnancement des nouvelles perspectives. Ces immeubles, et c'est là un élément important du modèle économique promu par Haussmann pour financer les travaux, furent conçus pour être des immeubles de rapport, l'entrée des appartements étant indépendante de celle des locaux du rez-de-chaussée, généralement voués à des activités professionnelles.

12 La trame haussmannienne s'appuie pour partie sur l'ancienne trame, héritée du Paris ancien. Mais elle la modifie profondément par les percées spectaculaires des nouveaux boulevards qui la structurent désormais, ainsi que par le dessin de grandes places, qui fonctionnent dans l'orientation spatiale des flux comme de véritables commutateurs. Si la démarche d'Haussmann était très pragmatique, elle n'en aboutit pas moins à créer une combinaison originale de trames en damier et, autour des places, de trames radiocentriques (Alonzo, 2005), conciliant sur un vaste territoire la compacité du bâti et l'acheminement rapide des personnes et des marchandises. Le modèle haussmannien inspira par la suite la transformation de nombreuses villes de province (Marseille, Lyon, Toulouse, Montpellier, Dijon, Angers, et bien d'autres).

\section{Les effets de la mécanisation des moyens de transport}

13 Les projets d'urbanisation de Cerdà et d'Haussmann sont marqués l'un et l'autre par leur forte cohérence. Pour Cerdà, les îlots en U, les carrefours à pans coupés, la trame en damier, et pour Hausmann, les immeubles de rapport, les places, les " percées » des grands boulevards, font «système ". C'est cette cohérence, associée à une maîtrise de l'occupation des sols, qui a assuré la pérennité et l'exemplarité de leurs démarches, très au-delà des contingences politiques dans lesquelles elles se sont inscrites.

14 Un élément n'avait cependant pas été complètement intégré par eux : les effets de la mécanisation des modes de transport. Au milieu du XIX ${ }^{e}$ siècle, le chemin de fer était déjà développé dans les liaisons interurbaines en Europe comme aux États-Unis. Les gares occupaient d'ailleurs une large place dans le projet haussmannien, et Cerdà avait quant à lui, à la suite dit-on d'un voyage à Paris, imaginé un transport ferroviaire souterrain. Mais la nouveauté fut que le chemin de fer fit progressivement irruption dans la ville, notamment avec le développement du tramway à traction électrique. Ce nouveau mode de transport inspira alors à la fois des réalisations et de nouvelles utopies, au premier rang desquelles il convient de citer celle imaginée à Madrid par Arturo Soria y Mata (1844-1920). Fort de son expérience de statisticien au sein de la compagnie des chemins de fer, il formula en 1882 un concept original de «cité 
linéaire », censé assurer une intégration parfaite entre l'urbain et le nouveau mode de transport.

«La structure des villes se transforme inconsciemment, instinctivement, en s'adaptant [...] aux progrès successifs des moyens de locomotion, car la forme des villes dérive de la forme de la locomotion [...]. Les villes devront prendre nécessairement les formes allongées et linéaires des voies ferrées » écrit Soria en 1913 dans « La cité linéaire, nouvelle architecture de villes $»^{1}$.

15 Sa proposition formulée pour le plan d'expansion de Madrid (figure 4) s'inscrit dans les mêmes problématiques que celles précédemment évoquées pour Barcelone et Paris: forte pression foncière, insalubrité, nécessité de répondre aux nouveaux besoins de mobilité. Pour ce faire, Soria imagine autour de la capitale espagnole une urbanisation en forme de ville linéaire qui s'étendrait sur $53 \mathrm{~km}$ le long d'une vaste artère de $500 \mathrm{~m}$ de largeur, et qui supporterait l'ensemble des infrastructures de transport (voie ferré et route) et des réseaux divers (eau, énergie, télégraphe,...). L'urbanisation se ferait autour de cet axe principal, organisée de part et d'autre en îlots orthogonaux répartis à intervalles réguliers, et rassemblant résidences, commerces, édifices publics, unités de production et espaces de loisirs... Le chemin de fer, dédié le jour aux déplacements de personnes, pourrait la nuit transporter des marchandises. Soria opte pour des densités faibles, selon les prescriptions des hygiénistes de l'époque, avec des maisons individuelles disposant de parcelles d'environ $400 \mathrm{~m}^{2}$ (Coudroy de Lille, 2002; El Hadeuf, 2016). Cet étalement est rendu possible par le nouveau mode de transport ferroviaire qui permet d'atteindre des vitesses jusqu'alors inespérées.

Figure 4 - Extension urbaine de la ville de Madrid, imaginée par A. Soria

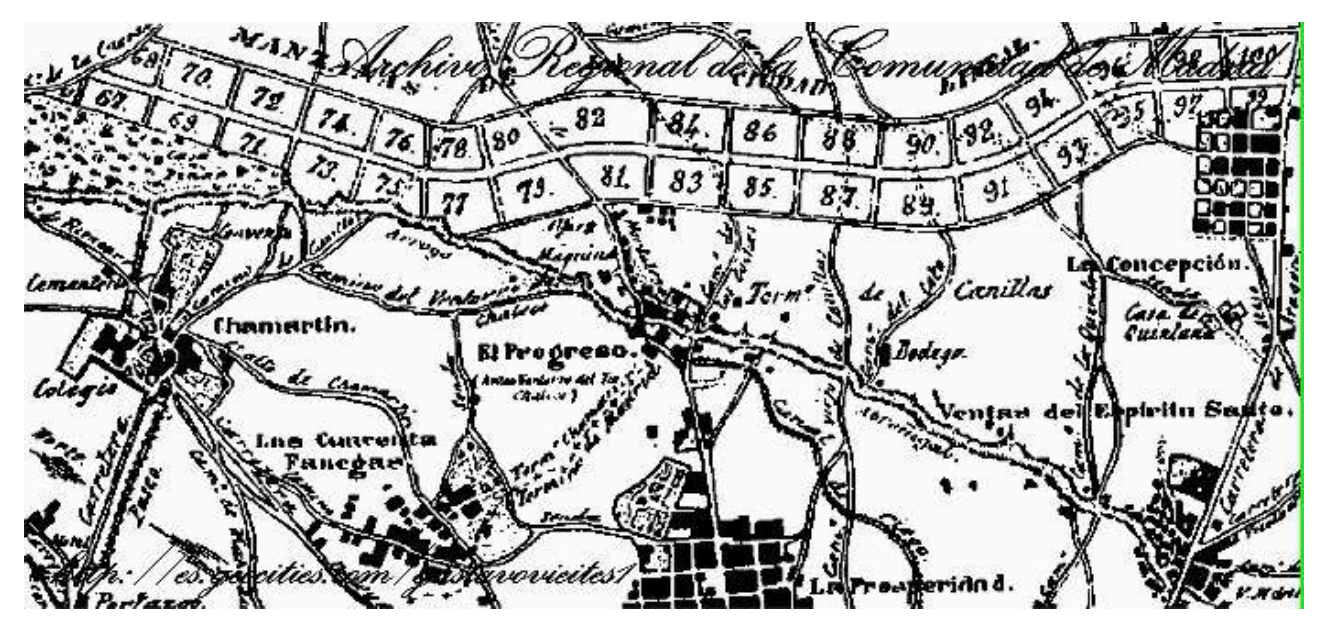

La cité linéaire, 1913

16 Le projet de Soria vit un début de réalisation, mais à une échelle beaucoup plus modeste que celle initialement envisagée : il s'étendit sur $5 \mathrm{~km}$, le long d'une voie de $40 \mathrm{~m}$ de large, qui relie le centre-ville de Madrid a sa banlieue Est. Seule, la taille des parcelles fut fidèle au projet initial, mais sa volonté de construire des habitations économiques resta en revanche utopique, et le quartier qu'il a conçu fit par la suite l'objet d'une importante spéculation.

17 On retrouve partiellement plus tard cette utopie dans «Illichville », ville imaginée dans les années quatre-vingt par un collectif d'artistes se réclamant de la pensée d'Ivan Illich, et plus près de nous et de manière beaucoup plus concrète, dans le concept de 
«ville creuse ». Celui-ci s'appuie sur une argumentation urbanistique et technique très fouillée (Maupu, 2005). Mais de tels projets, comme le montre de manière exemplaire l'évolution du quartier réalisé par Soria à Madrid, ont en commun de n'être envisageables que dans le cadre d'un contrôle très strict de l'usage des sols, difficilement compatible avec le fonctionnement de nos sociétés modernes.

On peut encore citer d'autres projets ou utopies urbaines, qui à la fin du XIX ${ }^{\mathrm{e}}$ siècle ou au début du XXe siècle, se réfèrent au développement du chemin de fer, notamment les projets de cité-jardins, dont Ebenezer Howard (1850-1928) fut un défenseur passionné. Fondateur en 1899 du mouvement des Garden Cities, il confia aux architectes Parker et Unwin le plan de Letchworth, et à Louis de Soissons celui de Welwyn, qui servirent après la Deuxième Guerre mondiale de prototypes pour les villes nouvelles de GrandeBretagne (Choay, 1965). Mais dans les projets de cités-jardins, contrairement au projet de cité linéaire de Soria, le chemin de fer apparaît plus comme un moyen de communication avec la ville-centre que comme un vecteur de structuration de l'espace urbain. Notons toutefois que, au début des années trente, le Conseil général du département de la Seine conçut un plan ambitieux de développement de cités satellites autour de Paris, inspiré du modèle des cités-jardins. Ce projet prenait appui sur le réseau de tramway sillonnant Paris et sa banlieue. Mais sous l'effet de la crise économique et du conflit entre la Ville de Paris et le Conseil général, qui se solda par le démantèlement du réseau de tramway, ce projet ne fut que très partiellement mis en œuvre (Larroque, 2002). L'« empreinte» des chemins de fer reste toutefois imprimée dans certains tissus urbains sous formes de "digitations », comme par exemple le long de la ligne de Sceaux, devenue aujourd'hui une branche du RER B.

Figure 5 - Le modèle de la ville-jardin d'E.Howard

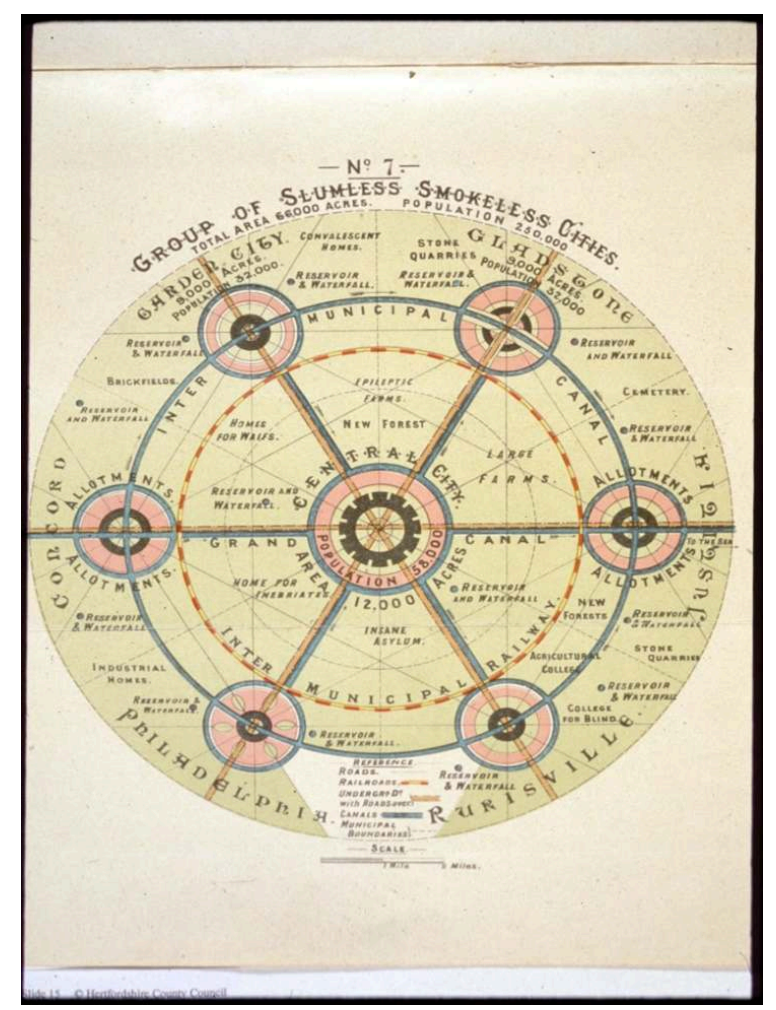

Garden Cities of Tomorrow, nouvelle édition, Londres, 1946 


\section{La ville de l'automobile} l'automobile comme mode dominant de déplacement urbain. Déjà, dans les années 1920-1930, les conflits d'occupation de l'espace entre les tramways et les automobiles s'étaient traduits par un repli des premiers dans de nombreux pays européens et par leur quasi disparition aux États-Unis et à Paris (les villes de province suivirent en France ce mouvement " de démontage » systématique des tramways, après la Seconde Guerre mondiale, à l'exception notable de la ville de Saint-Étienne). Mais la situation ne s'est pas stabilisée pour autant: les automobiles consomment en effet beaucoup d'espace, pour leur circulation et pour leur stationnement. Les places dessinées dans la trame des villes haussmanniennes, telles la place de la Concorde ou la place de l'Étoile à Paris, s'avérèrent en outre mal adaptées à la circulation d'un flux important de voitures et de camions : la trame radio-centrique présente en effet l'inconvénient de rabattre ces flux vers les points singuliers qu'elles constituent dans le réseau de voirie, et elles deviennent alors le lieu d'inextricables embouteillages, qui aboutirent de manière récurrente à une paralysie partielle du réseau. Dans ces conditions, l'entassement lié à la densité urbaine devint vite, pour les aménageurs, synonyme d'inefficience. L'évolution des modes de vie engendrant une demande de déplacements sans cesse croissante, cette remise en cause de la ville existante s'amplifia, non pas comme précédemment avec les cités-jardins au nom d'une redécouverte de la nature, mais à partir d'une approche fonctionnaliste.

Le courant dit de l' " urbanisme progressiste » accompagna largement ce mouvement et le dota d'un corpus théorique. Les valeurs mises en avant sont le progrès social et technique, l'efficacité et l'hygiène, et cela conduisit ce courant à proposer, pour l'extension urbaine, un espace standardisé et éclaté (Choay, 1965). À la ville haussmannienne, présentée comme cause de tous les maux et à sa mixité fonctionnelle (illustrée notamment dans la conception des immeubles de rapport qui induit une unité de lieu entre fonction résidentielle et activités commerciales), il va opposer un aménagement basé sur la spécification fonctionnelle des espaces (zonage). Les zones résidentielles sont alors séparées des zones d'activité et des zones de services ou de loisirs (centres commerciaux, salles multiplex, etc).

Cette démarche se fait au nom de la tranquillité des zones résidentielles et du respect du cadre de vie, et aussi au nom des réponses qu'il convient d'apporter aux désordres de la rue qui, dans la ville haussmannienne, est confrontée à de perpétuels conflits d'usage. L'architecture promue par l'urbanisme progressiste est elle-même profondément inspirée par cette démarche fonctionnaliste et plaide en faveur d'un large accès social à des appartements dotés du confort moderne, avec de grandes baies laissant généreusement pénétrer l'ensoleillement. Les ressources des techniques modernes, et notamment le béton armé, sont largement mobilisées à cet effet. Tony Garnier (1869-1948) a été l'un des principaux initiateurs de ce nouveau courant. Il imagina un projet révolutionnaire de cité industrielle moderne, qu'il présenta dès 1901, puis, agrémenté d'abondantes illustrations, en 1904. Dès cette époque, et avant même qu'il publie son ouvrage majeur ("Une cité industrielle », qui ne fut édité qu'en 1917), ses idées exercèrent une influence considérable (Choay, 1965). Elles lui valurent notamment d'exercer les fonctions d'architecte en chef de la ville de Lyon. Un autre

e-Phaïstos, V-1 2016 | 2018 
architecte emblématique de cette période est Walter Gropius (1883-1969), professeur à la célèbre école du Bauhaus, puis à Harvard. Mais le plus illustre représentant du courant progressiste reste Charles-Edouard Jeanneret (1887-1965) dit « Le Corbusier ", qui a animé avec son goût de la controverse les travaux des Congrès internationaux d'architecture moderne (CIAM) et est largement à l'origine de leur expression dans un document de référence, la Charte d'Athènes, publié en 1933. Pour lui, architecture et urbanisme sont inséparables, l'architecture moderne ne se conçoit pas sans ville moderne. Il reproche à la ville existante de ne pas avoir été animée d'un esprit de géométrie et affirme avec force la nécessité de principes fondamentaux auxquels il faut se tenir.

Figure 6. Le plan de Chandigarh imaginé par Le Corbusier (1951)

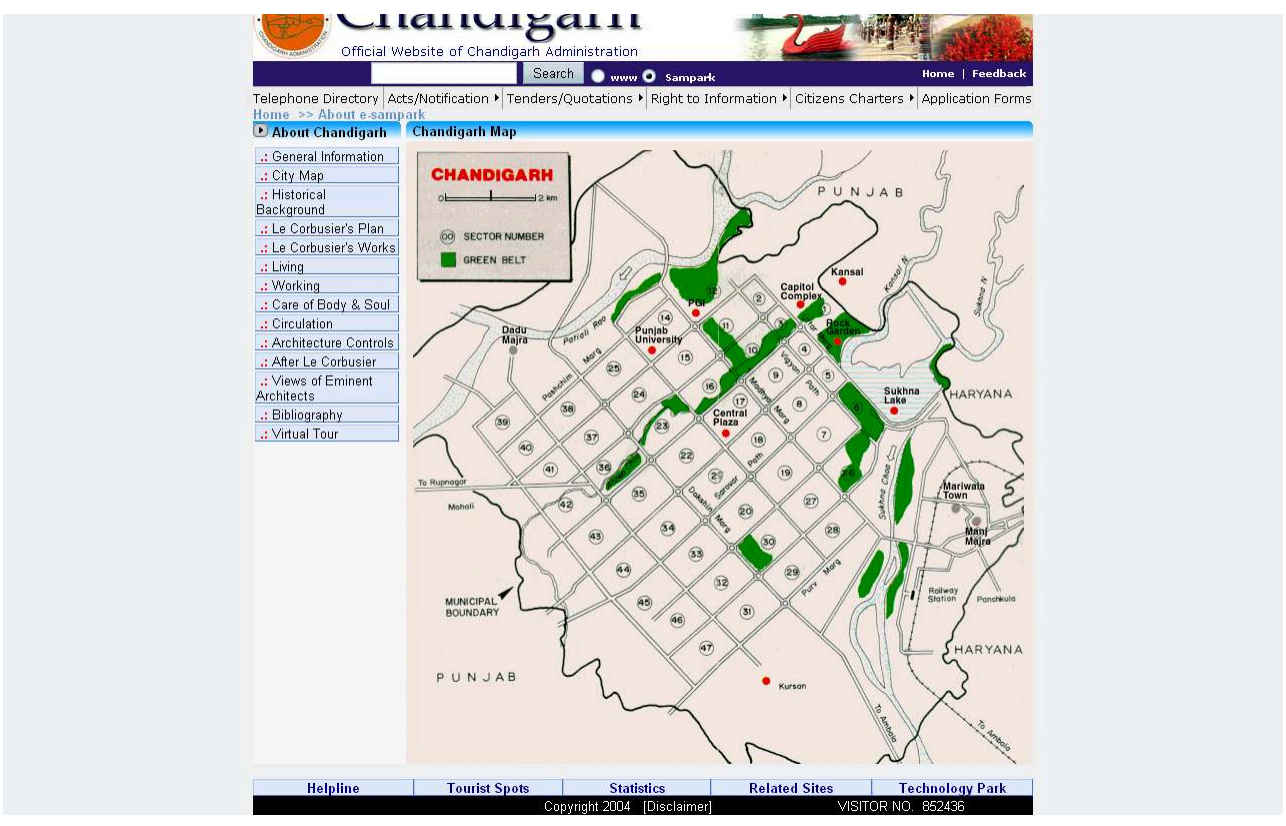

En matière de circulation, ces principes conduisirent Le Corbusier à proclamer la supériorité absolue de la ligne droite sur la ligne courbe : "La circulation exige la droite. La droite est saine aussi à l'âme des villes. La courbe est ruineuse, difficile et dangereuse ; elle paralyse $»^{2}$. Pour rendre la trame urbaine en damier plus efficace, il préconisa en outre une séparation stricte des trafics selon leur nature, allant jusqu'à identifier sept types de voirie ( règle des $7 \mathrm{~V} »$ ) selon la nature des flux et des modes (piétons, cyclistes, voitures, bus, poids lourds, etc), ces types de voirie pouvant se situer sur des niveaux différents. Le Corbusier en proposa trois : les poids lourds circulent en sous-sol, le trafic des voitures traversant la ville est quant à lui drainé par des autodromes portés par des passerelles de béton de 40 à $60 \mathrm{~m}$ de large, les piétons et les flux à usage local sont cantonnés au niveau intermédiaire, un système de dalles assurant la liaison entre ces différents niveaux. Ses théories trouvèrent leur expression la plus aboutie dans la ville de Chandigarh, capitale du Punjab en Inde, dont la réalisation lui fut confiée en 1951.

Les urbanistes progressistes ne sont pas les seuls à promouvoir l'avènement de la ville de la voiture. D'autres architectes-urbanistes prestigieux y contribuent également: parmi ceux-ci, une mention spéciale doit être attribuée à Frank Lloyd Wright (1869-1959). Contrairement aux théoriciens de l'urbanisme progressiste, son idée se 
caractérise par un refus de toute typologie au profit de la diversité, et sa vision est plus organique, voire naturaliste, que fonctionnaliste. Son apport à l'urbanisme se traduit principalement par le projet de «Broadacre City " qu'il formule entre 1931 et 1935 . Ce projet est basé sur la possibilité pour chaque famille de disposer d'une maison individuelle avec un terrain d'au moins un acre par personne, un réseau de communication très performant assurant une bonne accessibilité de tous les habitants à l'ensemble des services et aménités urbaines. Son plaidoyer en faveur d'une rencontre de la ville avec la nature, qui n'est pas sans rappeler celui d'E.Howard, se double d'un plaidoyer en faveur de la voiture individuelle, seule capable avec un réseau de voirie adapté, et notamment avec un réseau d'autoroutes urbaines, d'assurer cette « accessibilité généralisée ». «L'ère machiniste, écrit-il, ne nous a apporté (par rapport au Moyen Âge) aucune nouvelle forme de plan urbain [...]. Or aujourd'hui, le simple phénomène de l'automobile rend l'ancienne grande ville périmée ». Ou encore : «Le mouvement mécanique lié à l'automobile diffère absolument du mouvement de l'homme qui se déplace à pied ou utilise la traction animale. Ce nouvel étalon de mesure doit être appliqué à la conception générale de l'espace dans la planification de la cité nouvelle et de ses nouvelles demeures $»^{3}$.

Les théories de l'urbanisme progressiste et celles du naturalisme s'opposent sur beaucoup de points. Les premières revendiquent par exemple une certaine densité alors que les secondes prônent une sorte de dilution de la ville dans les espaces naturels. Mais elles se rejoignent dans la critique de la ville haussmannienne et dans une certaine assimilation entre urbanisation et étalement urbain, dont l'automobile devient un vecteur essentiel. Ces théories n'ont pas été acceptées sans susciter des controverses. Le cas du plan de reconstruction de Moscou, élaboré au début des années trente, est de ce point de vue tout à fait intéressant. E.Essaïan a conduit sur la genèse de ce plan un travail remarquable (Essaïan, 2006 et 2009); il met en évidence que, si l'influence d'Howard a pesé sur son élaboration notamment pour la conception des cités satellites de la périphérie, les thèses de Le Corbusier ont été en revanche critiquées et écartées ${ }^{4}$ par la Commission en charge de cette élaboration. Le choix après un débat approfondi d'un plan résolument radio-centrique, confortant le plan ancien, résulte à la fois de la volonté de préserver le centre de l'agglomération et sa consistance, et de le rendre le plus accessible possible en transport collectif pour les habitants des quartiers périphériques ${ }^{5}$. Peu cohérente avec cette démarche est en revanche la taille et la compacité des îlots, qui constituent dans cette optique autant d'obstacles pour les déplacements piétonniers.

Ces controverses ne doivent cependant faire oublier que les théories des urbanistes progressistes ont été plus souvent caricaturées que réellement mises en œuvre. La reconstruction de la ville du Havre sous la direction d'Auguste Perret (1874-1954) aux lendemains de la Deuxième Guerre mondiale témoigne de la possibilité de concilier réalisme et urbanisme progressiste : elle constitue un exemple tout à fait intéressant de réalisation d'une ville compacte, qui a su limiter l'étalement. D'abord critiqué par les amis de Le Corbusier, l'œuvre de Perret fut finalement reconnue et la ville du Havre constitue l'un des seuls ensembles architecturaux de l'époque moderne construits en Europe à être classé au patrimoine mondial de l'UNESCO. 


\section{La fin des utopies?} zonage qui sont reconnus et appliqués, notamment après la Seconde Guerre mondiale, dans un contexte où le manque de logements et l'expansion urbaine exercent une forte pression en faveur de solutions constructives efficaces et rapides à mettre en œuvre. Or sur le plan réglementaire et opérationnel, la délimitation de zones monofonctionnelles est un élément facilitateur appréciable (Dupuy, 1991). C'est l'époque où fleurissent les grands ensembles et les villes nouvelles. La nécessité de construire vite et beaucoup pèse par ailleurs sur les budgets et la trame urbaine est souvent réduite à sa plus simple expression : là où l'urbanisme progressiste militait pour une trame viaire en damier, on s'est contenté de réseaux arborescents qui ont contribué, la crise aidant, à transformer les grands ensembles en ghettos coupés du reste de la cité, ou, s'agissant des villes nouvelles, d'aménagements en grappes autour d'un axe routier, qui augmentent déraisonnablement les distances et la consommation d'espace. La séparation des fonctions s'est en outre accompagnée, sans que celle-ci ait toujours été recherchée, d'une aggravation des discriminations socio-spatiales. Elle s'est finalement traduite, à l'opposé des intentions proclamées, par un allongement moyen des déplacements pendulaires en distance, l'amélioration des performances des transports, notamment du fait d'un recours massif à l'usage de l'automobile pour les déplacements individuels, rendant compatible cet allongement avec le maintien de budgets-temps «quasiconstants ». De manière corrélative, la forte augmentation du trafic automobile s'est traduite par de nouvelles situations de congestion qui, si elles se sont déplacées des centres-villes vers la périphérie des agglomérations, n'en constituent pas moins ce contre quoi l'étalement urbain était censé nous prémunir... Bref, le constat a été fait que ce type de développement urbain ne pouvait pas être durable et qu'un changement de cap s'imposait.

Le rapport Buchanan a constitué au début des années soixante une première inflexion en faveur de la prise de conscience de cette nécessité (Buchanan, 1963). Il souligne notamment, prenant le contre-pied de l'urbanisme progressiste qui tendait à séparer la conception du cadre bâti et celle des infrastructures, qu' « il faut abandonner l'idée que les quartiers urbains se composent d'édifices disposés le long de voies de communication et comportent deux types de planification, l'un concernant les bâtiments et l'autre les rues [...]. Si les édifices et les voies d'accès sont conçus ensemble, dans une même démarche qui en font conjointement la substance de nos villes, ils pourront alors être intégrés selon des structures diverses, dont un grand nombre se révéleront beaucoup plus avantageuses que la rue classique ».

D'autres approches franchirent un pas supplémentaire dans la recherche d'une articulation transport / aménagement en préconisant un passage de la ville de l'automobile à la ville des transports collectifs, et parmi celles-ci, une approche qui s'est structurée autour du concept de "Transit Oriented Development» (TOD). Ce concept vise à orienter le développement urbain vers des configurations qui réduisent la dépendance automobile et s'appuient sur les transports collectifs en complémentarité des "modes doux ", et notamment de la marche. Pour ce faire, la démarche adoptée vise à coordonner les infrastructures et les services de transport collectif avec des projets urbains de moyenne et haute densité résidentielle, localisant des équipements publics, des emplois, des commerces et des services dans des quartiers à usage mixte.

e-Phaïstos, V-1 2016 | 2018 
Pour se référer à ce qui précède, on pourrait dire que ce concept s'inspire en la généralisant de la démarche proposée par Soria y Mata un siècle auparavant tout en prônant la mixité fonctionnelle de la ville haussmannienne, mais à une toute autre échelle, et en particulier, à une échelle intégrant l'aménagement des banlieues. La planification de l'usage des sols dans la ville de Curitiba au Brésil est souvent présentée comme une possible illustration du TOD. Robert Cervero (Cervero, 1997,2002) illustre ce concept par la règle des «3D»: Densité, Diversité fonctionnelle, Design, ce dernier terme concernant notamment le dessin des rues et des réseaux de transport public.

On peut reprocher à ce concept de raisonner sur une base mono-modale, là où la diversité des situations et l'hétérogénéité des espaces plaide en faveur de la plurimodalité, notamment entre la voiture et les transports collectifs. De ce point de vue, le parcours que nous venons de faire dans l'analyse des interactions entre trame urbaine et mode de transport invite à essayer de sortir du dilemme dans lequel évolue l'urbanisme depuis un siècle, qui tend à opposer ville de la voiture et ville des transports collectifs. La voie qui nous paraît tracée par l'analyse des enseignements de l'histoire urbaine nous amène à rechercher des combinaisons originales entre la trame en damier qui facilite l'écoulement du trafic, l'urbanisme linéaire en lien avec le développement des sites propres dédiés aux transports collectifs de surface, et l'urbanisme circulaire, autour des gares et des stations de métros (El Hadeuf, 2016).

En outre, le concept de TOD, si intéressant soit-il, ne résout pas le problème sur lequel ont déjà buté toutes les utopies précédemment évoquées: les infrastructures de transport, par l'accessibilité qu'elles procurent, créent de la valeur, laquelle risque fort de se trouver captée par des propriétaires fonciers, qui n'ont pas forcément l'obligation d'agir pour le bien collectif... Sauf dispositif spécifique, une infrastructure de transport urbain présente le paradoxe de constituer à la fois un lien entre les individus et les lieux, en même temps qu'un outil de différentiation spatiale et sociale. L'angle de vue ici choisi d'appréhender l'urbanisme à partir des trames qui structurent les systèmes urbains constitue une approche partielle d'un problème plus global et plus complexe auquel est confrontée la planification urbaine, qui est celui de la cohérence entre les objectifs fixés et les effets qu'ils produisent à différents horizons temporels. Renforcer cette cohérence demande des politiques publiques qui agissent dans la durée sur tout un ensemble de facteurs. Le "dessin » des trames viaires constitue un de ces facteurs, un facteur important mais un facteur seulement. C'est une condition nécessaire mais jamais suffisante.

Le rappeler est d'autant plus indispensable que les exigences aujourd'hui posées à la ville contemporaine au regard des enjeux du changement climatique doivent de plus en plus s'évaluer en terme de performances, et notamment de performances énergétiques. Est-ce pour autant la fin des utopies, ou bien ne convient-il pas plutôt de considérer que la ville durable constitue elle-même, au sens originel du terme, une utopie urbaine pour le XXI ${ }^{\mathrm{e}}$ siècle? 


\section{BIBLIOGRAPHIE}

Alonzo E., Du rond-point au giratoire, Parenthèses - Certu, Paris, 2005.

Benevolo L., Storia della città, traduit par C.Peyre, Parenthèses Ed., Marseille, 1983.

Buchanan C.D., Traffic in Towns, a study of the Long Term Problems of Traffic in Urban Areas, Londres, 1963.

Cervero R., Kockelman K., Travel Demand and the 3Ds: Density, Diversity and Design, in Transportation Research, part D, Vol 2, N³, 1997.

Cervero R., Ferrel C., Murphy S., Transit Oriented development and Joint Development in the United States: a litterature review, in transportation Cooperative Research program, report $n^{\circ} 52, D C$ Transportation Research Board, Washington, 2002.

Choay F., L'urbanisme, utopies et réalités, une anthologie, Seuil, 1965, Paris.

Coudroy de Lille L., Arturo Soria, urbaniste de la Cité linéaire, Urbanisme n³53, Mars-Avril 2007.

Dupuy G., L'urbanisme des réseaux, théories et méthodes, Armand Colin, Paris, 1991.

El Hadeuf M., L'interaction entre vitesse et morphologie urbaine : de la ville de l'automobile à la ville des transports collectifs, thèse, université Paris-Est, 2016.

Essaïan E., Le plan général de reconstruction de Moscou, La ville, l'architecte et le politique, Héritages culturels et pragmatisme économique, thèse en architecture, sous la direction de JeanLouis Cohen, Université Paris VIII, 2006.

Essaïan E., Portrait de Moscou, Cité de l'architecture, Paris, 2009.

Lhostis A., Le détour, la pause et l'optimalité : essai sur la distance et ses apports au transport et à l'urbanisme, thèse de HDR, Université Paris Est, 2015.

Larroque D., Margairaz M., Zembri P., Paris et ses transports, XIXe-Xxe siècle. Deux siècles de décision pour la ville et sa région », Ed.Recherches, 2002, Paris.

Lavedan P., Hugueney J., Henrat Ph., L'urbanisme à l'époque moderne, XVIè-XVIIIè siècles, Arts et Métiers Graphiques, 1982, Paris et Genève.

Maupu J.L., La ville creuse pour un urbanisme durable : nouvel agencement des circulations et des lieux, L'Harmattann, Paris, 2006.

Pawlowski K., Circulades languedociennes de l'An Mille, naissance de l'urbanisme européen, Presses du Languedoc, 2002.

Stipe M., Frank Lloyd Wrigth, Archives, Running Press Book Publisher, Philadelphia, traduit de l'Anglais par C.Perdereau, Ed. Française Seuil, Paris, 2004.

\section{NOTES}

1. Traduction de Georges Benoit Levy, rééditée en 1979, Paris, CERA.

2. Cité par F.Choay (Choay, 1965).

3. Cité par F.Choay (Choay, 1965)

4. Le Corbusier avait assorti le projet qu'il a proposé pour le Plan de Moscou d'une critique en règle du plan radio-centrique préexistant: «L'effort de l'urbaniste doit tendre à éviter 
l'institution d'une ville radiale et concentrique [...].Moscou est une ville provisoire, ne possédant pas une structure aussi indéniablement organisée que Paris, Londres, Berlin...» (cité par J.L.Cohen, Le Corbusier et la mystique de l'URSS, Mardaga Ed., Paris, 1995). La commission chargée de sélectionner les projets, présidée par V.Semionov, avait quant à elle regretté, s'agissant du projet de Le Corbusier, son «absence de compréhension de l'essence sociale des problèmes d'urbanisme qui se posent (aux autorités moscovites) » et considéré que son projet «relevait d'un schéma abstrait pour une ville idéale » (Essaïan, 2006).

5. Le projet finalement retenu consacre une place importante à l'organisation du système de transport. Il parle très peu en revanche de la circulation automobile, mais les prévisions de trafic $\mathrm{y}$ sont très modestes, ce qui semble bien indiquer qu'une augmentation importante de ce trafic n'était pas envisagée. Par contre coup, l'augmentation importante du nombre de véhicules en circulation depuis une décennie met en évidence le fait que la trame viaire n'a pas été prévue pour permettre une telle montée en charge et Moscou connait aujourd'hui des embouteillages endémiques.

\section{RÉSUMÉS}

Depuis deux millénaires, les trames urbaines rythment l'agencement des villes dans le temps long. De l'Antiquité et du Moyen-Âge, nous avons hérité de deux formes principales de trames, le damier et la forme radio-centrique. Au XIX ${ }^{e}$ siècle, la révolution industrielle nécessite d'apporter des réponses nouvelles à la forte croissance des flux de marchandises et de personnes. Les pères fondateurs de l'urbanisme moderne, Cerdà à Barcelone et Haussmann à Paris, se réfèrent à ces deux trames, quoique de manière très différente, tandis que Soria, inspiré par le succès du chemin de fer, plaide à Madrid en faveur d'un urbanisme linéaire. La promotion de la voiture individuelle à partir du début $\mathrm{du} \mathrm{XX}^{\mathrm{e}}$ siècle, largement prise en compte par les tenants de l'urbanisme progressiste, laisse un temps l'illusion d'un triomphe définitif et absolu de la trame en damier. Mais en réalité, ce triomphe est déjà contesté : le plan de Moscou, dans les années 1930 , est résolument radio-centrique. Et surtout, plus près de nous, la ville "du tout voiture » devient synonyme d'encombrement, de pollution, de consommation excessive d'espace et d'énergie. Aux États-Unis même, où la plupart des grandes métropoles illustrent jusqu'à la caricature l'urbanisme en damier, le concept de Transit Oriented Development (TOD) prône un retour à la ville des transports collectifs. Tout laisse aujourd'hui penser que la ville du futur devra combiner les atouts des différents modes de transport et pour cela, articuler dans la conception des réseaux de voirie l'urbanisme en damier qui facilite l'écoulement des flux, l'urbanisme linéaire en lien avec le développement des sites propres dédiés aux transports collectifs de surface, et l'urbanisme circulaire, autour des gares et des stations de métros.

For two millennia, urban patterns punctuate the layout of towns in long time. Of Antiquity and the Middel Ages, we inherited two main forms of patterns, the grid and radio-centric forms. In the $\mathrm{XIX}^{\mathrm{e}}$ century, the industrial revolution requires new solutions to face the important flows growth of goods and people. The founding fathers of modern urban planning, Cerdà in Barcelone and Haussmann in Paris, refer to these two patterns, although in a very different way. While Soria, inspired by the railway success, argues in favour of a linear urbanism in Madrid. The promotion of private cars since the early $\mathrm{XX}^{\mathrm{e}}$ century, which were largely taken into account by the proponents of progressive urbanism, achieve for a time the illusion of absolute and final 
triumph of the grid pattern. But in reality, this triumph is already contested: the Moscow plan in the 1930s is completely radio-centric. And above all, more recently, the "car oriented" city becomes synonymous of congestion, pollution, and excessive space and energy consumption. In the United States themselves, where the most major cities illustrate a caricature example of the grid pattern, the concept of Transit Oriented Development (TOD) advocates for a return to the transit city based on public transport. Today, everything leads to think that the city of tomorrow will have to combine the advantages of different transport modes, and that means, articulation between the grid road network design that facilitates the traffic flows, the linear urban pattern linked in the development of dedicated lanes for surface public transport, and circular urban planning around railway stations and subway stations.

\section{INDEX}

Mots-clés : histoire des techniques, trame urbaine, urbanisme, ville durable

Keywords : history of technology, urban pattern, urban planning, sustainable city

\section{AUTEUR}

\section{JEAN LATERRASSE}

Professeur émérite à l'Université Paris Est - Laboratoire Ville Mobilité Transport (UMR-T 94-3) 